\title{
Performance Analysis of Contending Customer Equipment in Wireless Networks
}

\author{
Humaira Afzal ${ }^{\mathrm{a}, *}$, Irfan Awan ${ }^{\mathrm{b}}$, Mohammed Rafiq Muftic ${ }^{\mathrm{c}}$, Ray E. Sheriff ${ }^{\mathrm{b}}$ \\ humairaafzal@bzu.edu.pk (Humaira Afzal), I.U.Awan@Bradford.ac.uk (I. Awan). rafiq_mufti@ciitvehari.edu.pk (M. Rafiq), \\ r.e.sheriff@bradford.ac.uk (R. E. Sheriff)
}

\begin{abstract}
a. Institute of Computing, Department of Computer Science, Bahauddin Zakariya University, Multan, Pakistan.
b. School of Electrical Engineering and Computer Science, University of Bradford, UK.

c. Department of Computer Sciences, COMSATS Institute of Information Technology, Vehari, Pakistan.
\end{abstract}

Keywords: Wireless network, customer premise equipment, initial contention window, initial ranging process, truncated binary exponential random backoff procedure.

\begin{abstract}
Initial ranging is the primary and important process in wireless networks for the customer premise equipments (CPEs) to access the network and establish their connections with the base station. Contention may occur during the initial ranging process. To avoid contention, the mandatory solution defined in the standards is based on a truncated binary exponential random backoff (TBERB) algorithm with a fixed initial contention window size. However, the TBERB algorithm does not take into account the possibility that the number of contended CPEs may change dynamically over time, leading to a dynamically changing collision probability. To the best of our knowledge, this is the first attempt to address this issue. There are three major contributions presented in this paper. First, a comprehensive analysis of initial ranging mechanisms in wireless networks is provided and initial ranging request success probability is derived based on number of contending CPEs and the initial contention window size. Second, the average ranging success delay is derived for the maximum backoff stages. It is found that the collision probability is highly dependent on the size of the initial contention window and the number of contending CPEs. To achieve the higher success probability or to reduce the collision probability among CPEs, the BS needs to adjust the initial contention window size. To keep the collision probability at a specific value for the particular number of contending CPEs, it is necessary for the BS to schedule the required size of the initial contention window to facilitate the maximum number of CPEs to establish their connections with reasonable delay. In our third contribution, the initial window size is optimized to provide the least upper bound that meets the collision probability constraint for a particular number of contending CPEs. The numerical results validate our analysis.
\end{abstract}

\section{Introduction}

Radio spectrum is a limited natural resource, the supply of which is now struggling to meet the seemingly ever increasing demand from consumers for mobile broadband data services. The reforming of spectrum, whereby bandwidth allocated to redundant or outmoded services is redeployed to new services is one means of providing bandwidth. This can now be seen in the allocation of bandwidth previously set aside for analogue TV to 4G mobile communications, following the introduction of digital TV services. The demand for mobile broadband data services may not simply be served by reallocating bandwidth, but may require some form of spectral sharing with incumbent services. A means of achieving this is through cognitive radio (CR), which builds on the concepts of Software Defined Radio put forward in the 1990s, whereby a service modifies its radio parameters within a given bandwidth, based on the transmission and reception environment in which it operates.

The IEEE 802.22 working group was formed in November 2004 to develop a first international standard based on a CR network. This was subsequently published in 2005 and accepted by the Federal Communication Commission. The basic purpose of this development was to provide broadband access in remote and rural areas by exploiting the unused TV band [1], [2], [3], [4]. Many other applications, such as real-time video streaming in home networks or automatic meter reading in the smart grid, can also be better supported. These systems can operate in the VHF/UHF, band ranging from $54 \mathrm{MHz}$ to $862 \mathrm{MHz}$ frequency, subject to non-interfering to the broadcast incumbents which may be digital or analog TV or wireless microphone. The main reason for TV band selection is twofold. The TV band is not always used in the geographical area of concern; it remains underutilized [5], [6], [7], [8], [9]. The second reason is of having lower frequencies compared to other licensed bands, therefore, resulting in lower propagation path loss [10]. Due to this feature, the spectral power density of the radio signal reduces slowly, which results in a high coverage area. The average coverage area is $33 \mathrm{~km}$ but it can be extended up to $100 \mathrm{~km}$, subject to weather condition, which is 
larger than IEEE 802.11 based Wi-Fi and IEEE 802.16 based WiMAX [11]. It is the first worldwide wireless standard based on CR techniques for the opportunistic use of TV band on a non-interfering basis. A CR observes its spectral environment and modifies its transmission parameters accordingly [11], [12], [13], [14], [15], [16]. A number of techniques can be incorporated in a CR network for the awareness of its environment. The two techniques used with wireless networks for spectral awareness are geo-location/database service and spectrum sensing [2]. In geo-location, the location information about each device is identified. The database service provides a list of available channels and their respective allowable effective isotropic radiated power (EIRP), which can be transmitted without providing any harmful interference to incumbent/licensed users in any given geographical area and is officially incorporated by the local regulatory authority. In a situation where no database service is required, all channels are assumed to be available. Spectrum sensing consists of observing the TV band and identifying which channels are not occupied by the licensed users. The wireless network operates on one of these unoccupied channels called operating channel and, in the case of licensed user occurrence, the entire network shifts quickly its transmission to the other free available channel [3], [17], [18].

The wireless network is based on a master/slave architecture with a single base station (BS) managing more than one customer premise equipments (CPE) through Medium Access Control (MAC). The BS controls all the communication in the cell, i.e., there is no peer-to-peer communication directly between the CPEs. The IEEE MAC is strictly connection-oriented, which requires CPEs to establish connections with the BS before any data transmissions can occur. Connection is the key element that can be identified by 12 bits in which 9 bits are reserved for station ID (SID) and 3 bits are for flow ID (FID). The station being under the control of BS is uniquely identified by SID, which can be unicast station (single CPE) or multicast station (group of CPEs), whereas FID represents a specific traffic flow assigned to a CPE. The combination of SID and FID defines a connection identifier (CID) for the particular CPE. Based on time division multiplex, data are scheduled in the downstream direction, while in the upstream direction the channel capacity is shared by CPEs on a demand basis according to an orthogonal frequency division multiplex (OFDMA) scheme. CPEs may lie at different locations in the large network; some are near while some are far away from the BS. Therefore, their transmission delays and received powers are different. To attain orthogonality among the sub-carriers in the upstream link, it is necessary that the signals generated by all the active CPEs must arrive at the BS synchronously. If not, CPEs will cause interference with each other and at the end BS will not be in a position to recover the individual signal of each CPE. Therefore, synchronization is an important aspect that can be achieved through a ranging process in which the CPEs adjust their timing offset and EIRP so that at the BS their transmitted signals having equal powers synchronized to the mini-slot boundary of the BS. The basic functions of this process at the BS comprise: timing estimation, ranging codes detection, and power estimation. The ranging process is further classified into initial ranging and periodic ranging. Initial ranging is the primary process for the CPEs to access the network and establish their connections with the BS. Work on the initial ranging process has been extensively discussed in [19], [20], [21], [22] for IEEE 802.11 and IEEE 802.16 but little work has been found in the literature for the IEEE 802.22 standard [23]. The contributions of this paper are threefold. Firstly, a comprehensive analysis of initial ranging mechanism is provided and initial ranging request success probability is derived based on a number of contending CPEs and the initial contention window size. Secondly, the average ranging success delay is derived for the maximum backoff stages. Since, the collision probability is highly dependent on the size of the initial contention window and the number of contending CPEs, therefore, to achieve the higher success probability, the BS needs to adjust the initial contention window size. To keep the collision probability at a specific level for the particular number of contending CPEs, it is essential for the BS to schedule the required size of the initial contention window to facilitate the maximum number of CPEs to establish their connections with reasonable delay. Our third contribution is to optimize the initial window size and to provide the least upper bound that meets the collision probability constraint for a particular number of contention CPEs. This paper is organized as follows: Section 2 provides an overview of IEEE 802.22 network. An analytical model is then derived in Section 3 and numerical results are discussed in Section 4 before concluding remarks are presented in Section 5.

\section{Overview of IEEE 802.22}

The wireless regional area network (WRAN) consists of one BS and a number of fixed or portable CPEs having different quality of service (QoS) requirements. The cell follows fixed-point to multi-point topology with a master/slave architecture and can facilitate up to $512 \mathrm{CPEs}$ after fulfilling the requirements necessary for the protection of the incumbent. No CPE is allowed to transmit without receiving appropriate authorization from a BS. 
The BS manages all the activities regarding modulation, coding etc. of its associated CPEs by MAC. There are two operational modes, i.e., normal mode and self-coexistence mode, defined in the IEEE 802.22 standard [2]. A WRAN cell works by default in normal mode and switches to self-coexistence mode if another WRAN cell is detected. This paper is focused on normal mode. During normal operation, a WRAN cell broadcasts a superframe preamble and a superframe control header $(\mathrm{SCH})$ at the beginning of every superframe on its operating channel. Each superframe has a duration of $160 \mathrm{~ms}$ and is composed of 16 frames, each having a length of $10 \mathrm{~ms}$. All transmissions can be organized into downstream (DS) and upstream (US) transmissions based on the direction of transmissions. The DS transmission is defined from the BS to CPE. Conversely, the US transmission is in the opposite direction. According to the IEEE 802.22 standard, the BS is responsible for scheduling both DS and US transmissions. All scheduling behavior is put into the MAC frame. The structure of a MAC frame can be divided into DS subframe and US subframe. The DS subframe is for DS transmissions. Similarly, the US subframe is for US transmissions. The first MAC frame contains superframe preamble, frame preamble, $\mathrm{SCH}$, frame control header $(\mathrm{FCH})$ and data payload as shown in Figure 1. Its payload is reduced by two symbols to compensate for the superframe preamble and SCH.

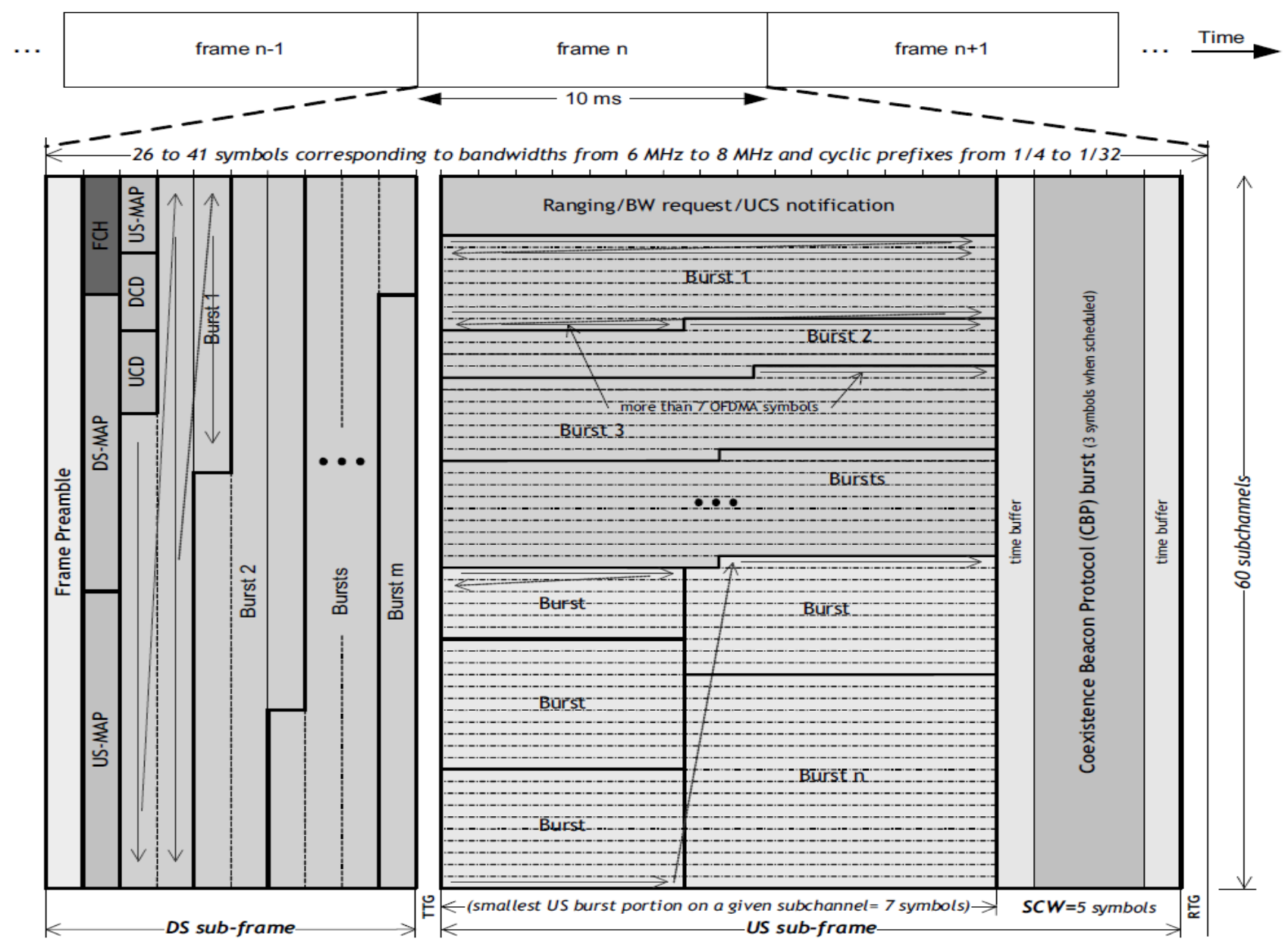

Figure 1: The frame structure in IEEE 802.22 standard.

The superframe preamble showing at the beginning of the superframe is for frequency and time synchronization purposes. The SCH carries the BS MAC address and other information necessary for the CPE to initiate a connection. To protect incumbents and to handle a self-coexistence situation, sufficient mechanism is included in the SCH. The quiet period schedule for sensing is also given. Frame preamble indicates the beginning of the frame. It is used for synchronization, channel estimation, frequency offset estimation and received power estimation. FCH contains information about the frame and is transmitted as a part of DS-PDU. Further, DS subframe contains DS-MAP, USMAP, upstream channel descriptor (UCD), downstream channel descriptor DCD etc. The DS-MAP and US-MAP are used respectively to indicate downstream and upstream subframe structures. The transmission parameters of possible upstream channels are contained in UCD, whereas DCD has characteristics of possible downstream channels. As shown in Figure 1, the DS subframe consists of only one PHY PDU but US subframe is composed of more than one PHY PDU, with each transmitted from a different CPE. In addition, US subframe has contention windows for initial ranging, periodic ranging, bandwidth request, Urgent Coexistence Situation (UCS) notification and Self-Coexistence 
situation. Contention may occur during initial ranging, periodic ranging, bandwidth request etc. To avoid contention during initial ranging, Code Division Multiple Access (CDMA) is specified in IEEE 802.22 WRAN standard. To perform initial ranging, the CPE first acquires downlink synchronization and uplink transmission parameters from DSMAP and US-MAP, respectively. Then it selects a number from its internal backoff window and a CDMA code from the given available codes using a uniform random process. The CPE then modulates the CDMA code with the initial ranging channel in the selected time slot to the BS. The ranging channels assigned by the BS are indicated by the number of subchannels in US-MAP. If a CPE selects a number 10 from its internal backoff window, it having values of between 0 and 15, then it must defer a total of 10 contention transmission opportunities before a transmission attempt (RNG-REQ). If the first US-MAP is for 4 transmission opportunities, the CPE does not utilize this and requires 6 more transmission opportunities to defer. If the second US-MAP is for 5 transmission opportunities, the CPE again does not use this and needs one more transmission opportunity to defer. If the next US-MAP is for 7 transmission opportunities, the CPE transmits RNG-REQ on the 2nd transmission opportunity after deferring the first one.

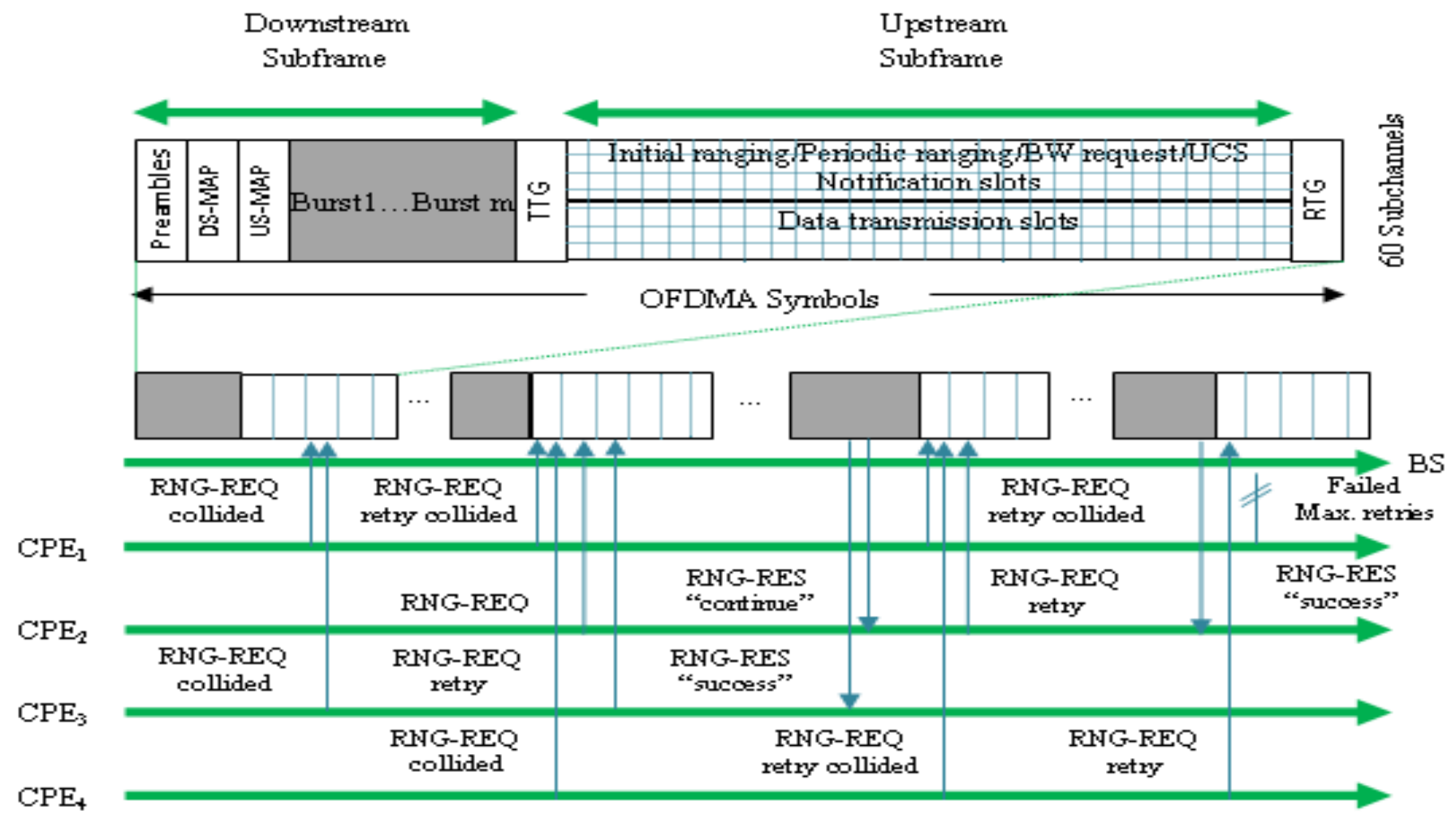

Figure 2: Initial ranging illustration.

After transmitting the RNG-REQ, the CPE has to wait for a BS response (RNG-RSP). The decision on whether a transmission of RNG-REQ is successful or not is made by the CPE in accordance with the information contained in RNG-RSP. According to the IEEE 802.22 standard, if CPE does not receive any feedback from BS in the subsequent US-MAP, the CPE assumes that the transmission failed due to collision with other RNG-REQs. Note that collisions can occur when two or more CPEs want to be associated with the BS and send their request on the same ranging slot on the same channel as shown in Figure 2. In this case, the CPE enters into the next retrial stage using truncated binary exponential backoff mechanism, by increasing its backoff window size by a factor of two, as long as it is less than the maximum backoff window size. Using this new backoff window, the CPE then selects a number randomly and repeats the above process. There exists some upper bound in the number of retries for association with the BS. When this bound is achieved by the particular CPE, the used downlink channel is marked as unusable and the CPE will start scanning for another downlink channel. After successfully receiving the RNG-REQ, the BS extracts information regarding timing and power. Then it broadcasts the RNG-RSP message that contains the received ranging code, the ranging time slot and a status notification. This response message is identified by the relevant CPE. The status notification may be success or continue. In case of continue, the RNG-RSP message contains additional information about timing and power adjustments. The CPE considers this transmission attempt unsuccessful, and after making the suggested modifications (power level, timing adjustments), it sends another RNG-REQ in the initial 
ranging slot using random backoff. If the status in RNG-RSP message is success, the ranging process will be completed. This entire ranging process is illustrated in the following pseudocode-1.

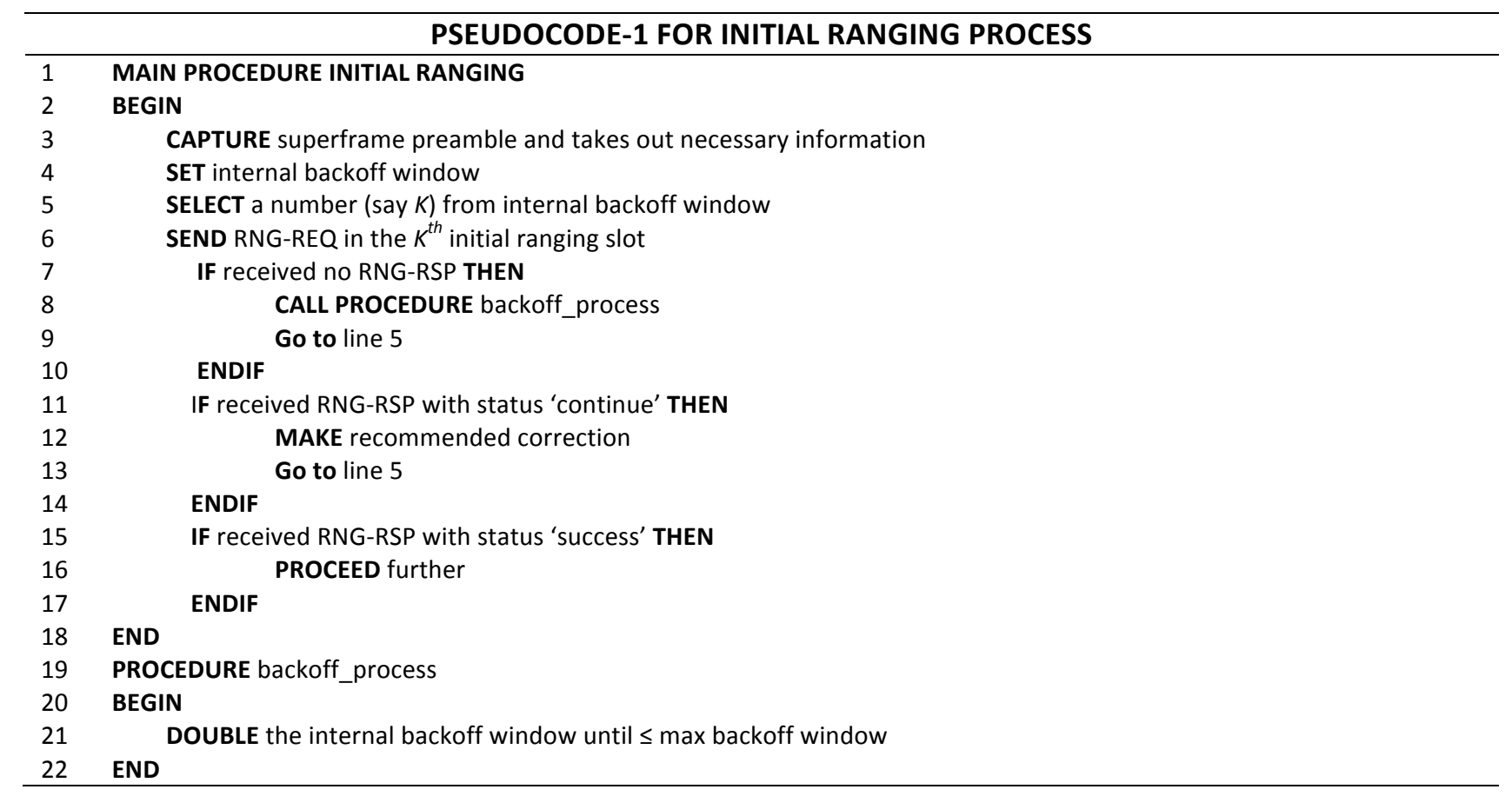

\section{Analytical Model}

In this section, we derive the expression for initial ranging request collision probability, ranging request success probability and average ranging success delay, in terms of number of contention CPEs and initial contention window size. The analysis is based on a probabilistic framework utilizing two dimensional transition diagram. Without loss of generality, the following assumptions are used in our model.

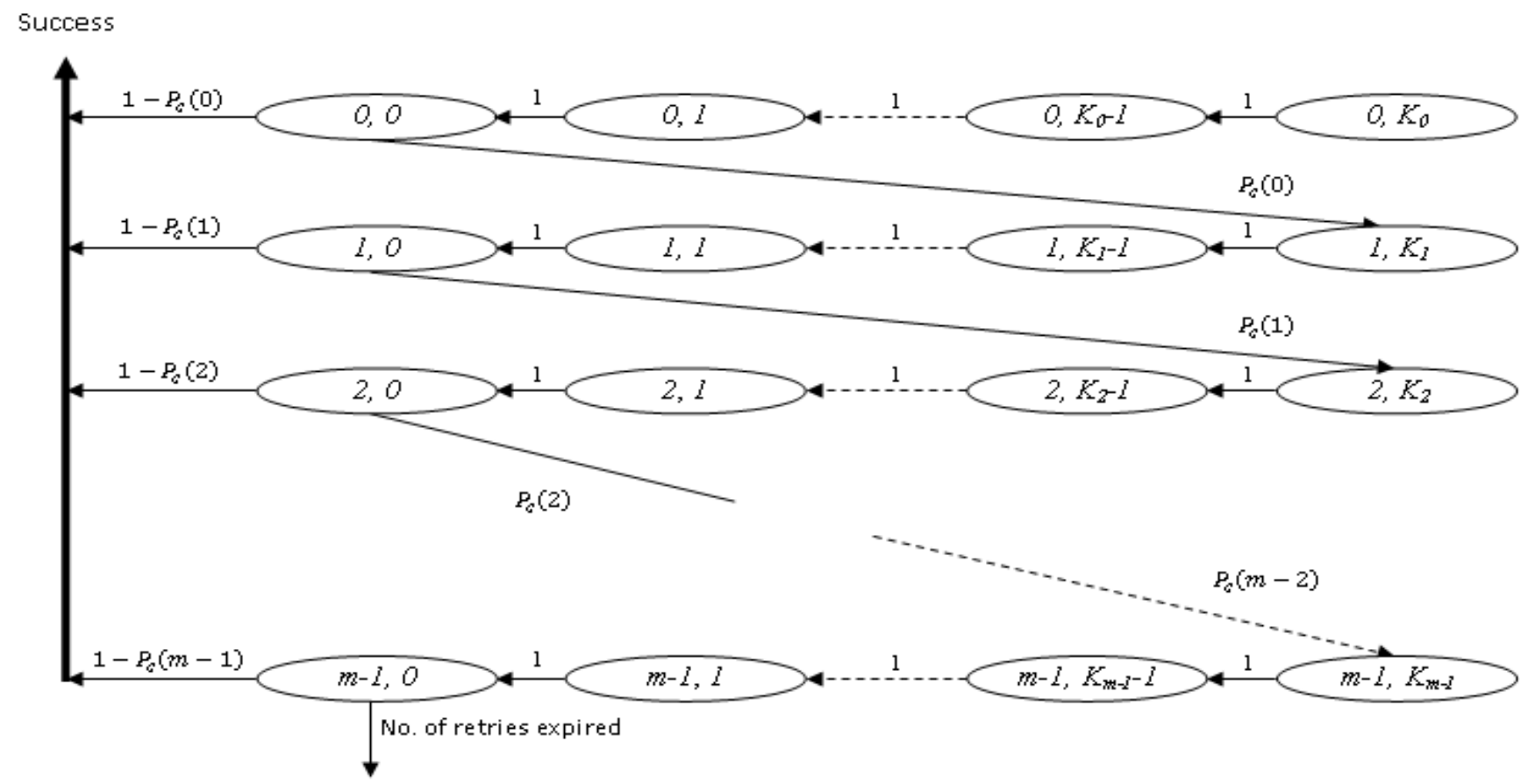

Figure 3: Probability transition model for IEEE 802.22 backoff process.

- Each CPE has geolocation information acquiring capability and sensing capability.

- Each CPE updates the required parameters correctly after the BS recommendations.

- When BS successfully receives the ranging request without requiring any modification, it will associate the CPE with the network. 
- During initial ranging process if an incumbent is detected, the CPE will not proceed further on the operating channel according to spectrum etiquettes [2]. We assume that no incumbent will occur on the operating channel during this process.

\subsection{Initial ranging request success probability}

For simplicity, we call initial ranging request success probability a ranging success probability $\left(P_{S}().\right)$. This probability indicates that the initial ranging request (RNG-REQ) has successfully reached the BS. Based on the given assumptions, the BS will associate the requested CPE with its network after successfully receiving the ranging request. To derive $P_{S}($.$) , we proceed as follows. Let C$ be a stochastic process representing a counter and $B(t)$ be a random process representing a backoff stage of the given CPE at time $t$. The maximum number of backoff stages for each attempted CPE is $m$, where $m$ is an integer such that $m>0$. Each stage has a backoff window corresponding to the states at that stage. Let $W_{j}=2^{j} W_{0}$ where $j \in\{0,1,2, \ldots m-1\}$ be the internal backoff window incorporated by CPE for the $j^{\text {th }}$ backoff stage. The CPE selects a random number $K_{j}$ from the set $\left\{0,1, \ldots W_{j}-1\right\}$ such that $0 \leq K_{j}<W_{j}$ and suppose that $C_{W_{j}}=K_{j}$ and its value is decremented by deferring one transmission opportunity given in the US-MAP. When $C_{W_{j}}=0$, the CPE will transmit its initial ranging request (RNG-REQ) in the available transmission opportunity on the initial ranging channel. It is also possible that there is more than one CPE whose $C_{W_{j}}$ reaches zero at the same time, which means that there are more than one CPE trying to send a RNG-REQ in the same transmission opportunity. In this situation, collision occurs. Let $P_{c}(j)$ be the probability that the initial ranging request experiences a collision at the $j^{\text {th }}$ backoff stage. Now consider the two dimensional probability transition diagram shown in Figure 3, i.e., $\left(B(t), K_{j}\right)$, where $B(t) \in\{0,1,2, \ldots m-1\}$ and $j \in\{0,1,2, \ldots m-1\}$. Transitions take place between states in every stage with probability 1 .

The transition probabilities can be presented as follows:

$$
\begin{aligned}
& P\{(x, y-1) \mid(x, y)\}=1 \text {, where } x \in\{0,1, \ldots m-1\} \text { and } y \in\left\{1,2, \ldots K_{j}\right\}, K_{j}=C_{W_{j}} \text { and } j \in\{0,1, \ldots m-1\} \\
& P\{(x, 0)\}=1-P_{c}(j), \text { where } x \in\{0,1, \ldots m-1\} \text { and } j \in\{0,1, \ldots m-1\} \\
& P\left\{\left(x+1, K_{j}\right) \mid(x, 0)\right\}=P_{c}(j), \text { where } x \in\{0,1, \ldots m-2\} \text { and } K_{j}=C_{W_{j}} \text { and } j \in\{0,1, \ldots m-1\}
\end{aligned}
$$

When $C_{W_{j}}=0$, the probability that CPE successfully sends the initial ranging request on the transmission opportunity is $1-P_{c}(j)$. It is also possible that the BS successfully receives the RNG-REQ but rejects it due to not fulfilling its timing and power requirements. Let $q$ be the probability of the BS that the RNG-REQ of the CPE is rejected and is required to be repeated after some adjustments. In this case, the CPE will select a fresh random number $K_{j}$ from the set $\left\{0,1, \ldots W_{j}-1\right\}$ and will repeat the ranging process again. So the probability of ranging request failure is primarily concerned with these two main factors: collision and rejection by the BS. This probability at the $j^{\text {th }}$ backoff stage, which is denoted by $P_{F}(j)$, can be represented as

$P_{F}(j)=P_{c}(j)+\left(1-P_{c}(j)\right) q$

Since CPE is assumed to be a capable device so it is reasonable that $\mathrm{q}$ is extremely small such that $\mathrm{q} \approx 0$, therefore

$P_{F}(j)=P_{c}(j)$

Let $n$ be the total number of contended CPEs (previously contended CPEs and current attempted CPEs). It is assumed that $n$ should be smaller than or equal to the difference of total capacity $(N)$ of an IEEE 802.22 WRAN cell and its number of already associated CPEs $(A)$, i.e. $n \leq N-A$. Collisions will take place when at least two CPEs select the same transmission opportunity. Therefore, given $n$ CPEs, the ranging request collision probability at $j^{\text {th }}$ backoff stage can be derived as:

$$
P_{c}(j)=\left\{\begin{array}{c}
0 \quad \text { if } n<2 \\
\sum_{\phi=2}^{n}\left(\begin{array}{c}
n \\
\phi
\end{array}\right) p_{j}^{\phi}\left(1-p_{j}\right)^{n-\phi} \quad \text { if } n \geq 2 \\
\text { where } p_{j}=\frac{1}{W_{j}}, W_{j}=2^{j} W_{0}, \text { for } j=0,1,2, \ldots m-1
\end{array}\right.
$$


Now finally, the probability of ranging success, denoted as $P_{S}(j)$, is written as:

$P_{S}(j)=1-P_{F}(j)=1-P_{c}(j)$ where $j \in \mathbb{Z}$ and $0 \leq j \leq m-1$

Now, substituting (6) into (7), we can obtain the ranging success probability at the $j^{\text {th }}$ backoff stage.

\subsection{Derivation of least upper bound on initial contention window size $\left(W_{0}\right)$}

Now we are interested in determining the minimum initial contention window size at a specific collision probability level for a particular number of contending CPEs. From equation (6), after incorporating the value of $p_{j}$ into this expression we have:

$$
\begin{aligned}
& P_{c}(j)=\sum_{\phi=2}^{n}\left(\begin{array}{l}
n \\
\phi
\end{array}\right)\left(\frac{1}{W_{j}}\right)^{\phi}\left(1-\frac{1}{W_{j}}\right)^{n-\phi} \\
& P_{c}(j)=\sum_{\phi=2}^{n}\left(\begin{array}{l}
n \\
\phi
\end{array}\right)\left(\frac{1}{W_{j}}\right)^{\phi}\left(\frac{W_{j}-1}{W_{j}}\right)^{n}\left(\frac{W_{j}}{W_{j}-1}\right)^{\phi} \\
& P_{c}(j)=\left(\frac{W_{j}-1}{W_{j}}\right)^{n}\left[\sum_{\phi=0}^{n}\left(\begin{array}{l}
n \\
\phi
\end{array}\right)\left(\frac{1}{W_{j}-1}\right)^{\phi}+-1-\left(\frac{n}{W_{j}-1}\right)\right] \\
& P_{c}(j)=\left(\frac{W_{j}-1}{W_{j}}\right)^{n}\left[\left(\frac{W_{j}}{W_{j}-1}\right)^{n}+-1-\left(\frac{n}{W_{j}-1}\right)\right] \\
& P_{c}(j)=1-\left(\frac{W_{j}-1}{W_{j}}\right)^{n}-n\left(\frac{W_{j}-1}{W_{j}}\right)^{n}\left(\frac{1}{W_{j}-1}\right)
\end{aligned}
$$

Let $P_{c}(j)<\Omega$ where $\Omega \in[0,1]$, then equation (12) can be written as:

$1-\left(\frac{W_{j}-1}{W_{j}}\right)^{n}-n\left(\frac{W_{j}-1}{W_{j}}\right)^{n}\left(\frac{1}{W_{j}-1}\right)<\Omega$

$-\left(\frac{W_{j}-1}{W_{j}}\right)^{n}-n\left(\frac{W_{j}-1}{W_{j}}\right)^{n}\left(\frac{1}{W_{j}-1}\right)<\Psi$

where $\Psi=\Omega-1, \Psi \in \mathbb{R}$

$\frac{\left(W_{j}-1\right)^{n-1}\left(W_{j}-1+n\right)}{W_{j}^{n}}>\Psi$

Finally, $\quad W_{j}<\sqrt[n]{\frac{\left(W_{j}-1\right)^{n-1}\left(W_{j}-1+n\right)}{\Psi}}$

where $W_{j}=2^{j} W_{0}$, for $j=0,1,2, \ldots m-1$

This expression gives the general formula for finding the least upper bound on initial contention window size for $n$ number of contention CPEs with the constraint $P_{c}(j)<\Omega$ where $\Omega \in[0,1]$.

\subsection{Backoff delay derivation}

Suppose CPE is powered on and it captures the superframe preamble and extracts the necessary information regarding minimum backoff window, maximum backoff window, synchronization parameters, transmission parameters etc. The window sizes are controlled by the BS and are stipulated in the DS-MAP. These sizes are expressed as a power of 2 value. For example, a value of 5 indicates that the contention window size is 32 . The CPE sets its internal backoff window according to the minimum backoff window allocated by the BS. Let this internal backoff window be $W_{0}$. The CPE then randomly selects a number from the set $\left\{0,1, \ldots W_{0}-1\right\}$. Let this number be $K$. The CPE has to defer a total of $K$ initial ranging opportunities (ROs)/slots over multiple frames before transmitting the initial ranging request (RNG-REQ). These ROs are given in US-MAP. Each frame has one or more ROs. We assume that each frame 
has a fixed number of ROs. Therefore, the number of frames that the CPE has to defer is $\frac{K}{R O S}$. In this case the delay $\left(D_{0}\right)$ will be $\frac{K}{R O S} *$ Frame duration. Further, the CPE needs to defer $(K \bmod R O s)$ initial ranging slots along with $\frac{K}{R O S}$ frames. Therefore, $D_{0}$ can be written as:

$D_{0}=\frac{K}{R O S} *$ Frame duration $+(K \bmod R O s) *$ slot duration

For simplicity, Frame duration $=T_{F D}$ and slot duration $=T_{R O}$, Now Equ (17) takes the form as

$D_{0}=\frac{K}{R O S} * T_{F D}+(K \bmod R O S) * T_{R O}$

Since $K$ varies from 0 to $W_{0}-1$, therefore we take the average value. Finally, $D_{0}$ can be written as:

$D_{0}=\frac{\sum_{K=0}^{W_{0}-1}\left(\frac{K}{R O S} * T_{F D}+(K \bmod R O S) * T_{R O}\right)}{W_{0}}$

Let $P_{c}(j)$ be the probability that the ranging request experiences a collision, i.e. failed transmission then $1-P_{c}(j)$ is the probability that the ranging request is transmitted successfully. In case of collision, the CPE will double the size of its internal backoff window. Let the new window be $W_{1}$. Now the CPE will randomly select the number $K$ from $\left\{0,1, \ldots W_{1}-1\right\}$. Similarly, delay $\left(D_{1}\right)$ can be computed as follows:

$D_{1}=\frac{\sum_{K=0}^{W_{1}-1}\left(\frac{K}{R O S} * T_{F D}+(K \bmod R O S) * T_{R O}\right)}{W_{1}}$

If collision again occurs, the CPE will involve in the third backoff phase and adopt the same process, i.e., it will double the size of internal backoff window. Let us assume that the CPE completes its successful ranging request in a maximum of $\mathrm{m}$ attempts or backoff phases. Therefore, we have the following delays for the remaining backoff phases.

$$
\left[\begin{array}{c}
D_{2} \\
D_{3} \\
\cdot \\
\cdot \\
D_{m-1}
\end{array}\right]=\left[\begin{array}{c}
\frac{\sum_{K=0}^{W_{2}-1}\left(\frac{K}{R O S} * T_{F D}+(K \bmod R O s) * T_{R O}\right)}{W_{2}} \\
\frac{\sum_{K=0}^{W_{3}-1}\left(\frac{K}{R O S} * T_{F D}+(K \bmod R O s) * T_{R O}\right)}{W_{3}} \\
\cdot \\
\frac{\sum_{K=0}^{W_{m}-1}\left(\frac{K}{R O S} * T_{F D}+(K \bmod R O s) * T_{R O}\right)}{W_{m-1}}
\end{array}\right]
$$

Where $W_{1}=2 W_{0}, W_{2}=2 W_{1}, W_{3}=2 W_{2}, \ldots \quad W_{m-1}=2 W_{m-2}$

Now we consider the worst case where the ranging request obtains success in the $\mathrm{m}^{\text {th }}$ phase, so there will be $(m-1)$ failures. The resulting delay $(D)$ can be computed as:

$D=\left(1-P_{c}(0)\right)\left[D_{0}+t_{s}\right]+P_{c}(0)\left(1-P_{c}(1)\right)\left[D_{0}+t_{c}+D_{1}+t_{s}\right]+P_{c}(0)^{2}\left(1-P_{c}(2)\right)\left[D_{0}+t_{c}+D_{1}+t_{c}+D_{2}+\right.$ $\left.t_{s}\right]+P_{c}(0)^{3}\left(1-P_{c}(3)\right)\left[D_{0}+t_{c}+D_{1}+t_{c}+D_{2}+t_{c}+D_{3}+t_{c}\right]+\cdots+P_{c}(0)^{m-2}\left(1-P_{c}(m-1)\right)\left[D_{0}+t_{c}+\right.$ $\left.D_{1}+t_{c}+D_{2}+t_{c}+\cdots+D_{m-1}+t_{s}\right]$

Where $t_{c}$ is the time when failure is assumed due to not receiving any response from the BS which is equal to the one frame duration in IEEE 802.22 network and $t_{s}$ is the time when status notification success is received in the next USMAP. Since $t_{c}=t_{s}=t$, so Equ (22) can be formulated as: 


$$
\begin{aligned}
D=\left(1-P_{c}(0)\right) & {\left[D_{0}+t\right]+P_{c}(0)\left(1-P_{c}(1)\right)\left[\sum_{j=0}^{1} D_{j}+2 t\right]+P_{c}(0)^{2}\left(1-P_{c}(2)\right)\left[\sum_{j=0}^{2} D_{j}+3 t\right] } \\
& +P_{c}(0)^{3}\left(1-P_{c}(3)\right)\left[\sum_{j=0}^{3} D_{j}+4 t\right]+\cdots+P_{c}(0)^{m-1}\left(1-P_{c}(m-1)\right)\left[\sum_{j=0}^{m-1} D_{j}+m t\right]
\end{aligned}
$$

The expression given in Equ (23) gives the average delay when the ranging request is achieved in $m^{\text {th }}$ backoff stages. Now for simplicity, let us consider the particular case when no backoff is required i.e., $m=0$ and we investigate the average ranging success delay over different values of initial contention window size.

Now, by Equ (23) with $m=0$, we have:

$D=\left(1-P_{c}(0)\right)\left[D_{0}+t\right]$

$D\left(n, W_{0}\right)=\left[1-\sum_{\phi=2}^{n}\left(\begin{array}{l}n \\ \phi\end{array}\right)\left(\frac{1}{W_{0}}\right)^{\phi}\left(1-\frac{1}{W_{0}}\right)^{n-\phi}\right] \times\left[\frac{\sum_{K=0}^{W_{0}-1}\left(\frac{K}{R O S} * T_{F D}+(K \bmod R O s) * T_{R O}\right)}{W_{0}}+t\right]$

\section{Numerical results and discussions}

To evaluate the mathematical model developed in the previous Section, we numerically solve expressions given in Equs (6)-(7) and Equ (25) to calculate the value of $P_{c}(j), P_{S}(j)$ and $D\left(n, W_{0}\right)$. The parameters used in the numerical results are given as:

$R O S=$ Initial ranging request opportunities per frame $=5$

$T_{F D}=$ Frame duration in IEEE 802.22 network $=10 \mathrm{~ms}$

$T_{R O}=$ Transmission slot duration in IEEE 802.22 network $=0.624 \mathrm{~ms}$

$t=B S$ response time $=$ One frame duration $=10 \mathrm{~ms}$

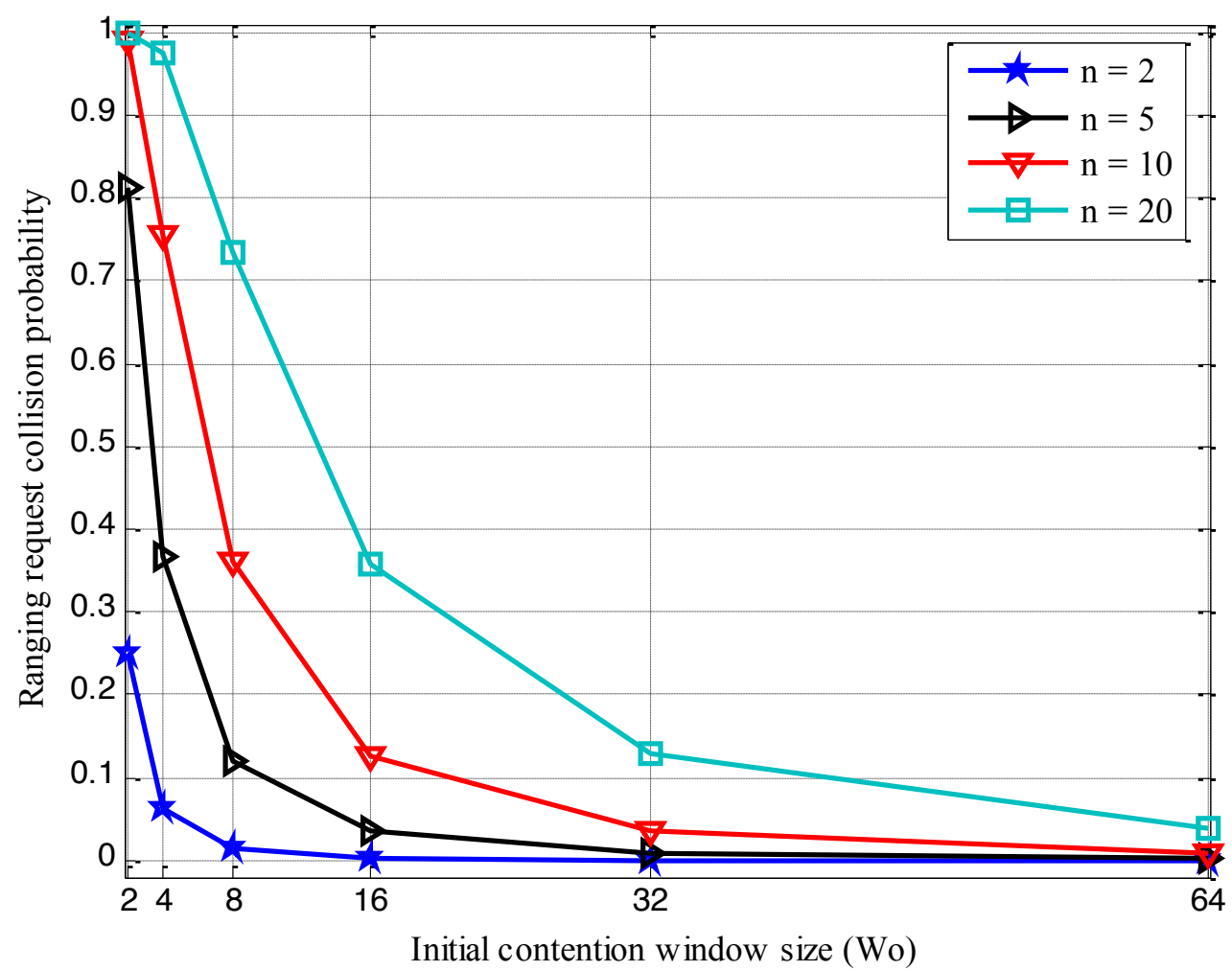

Figure 4: Ranging request collision probability versus initial contention window size. 
Figure 4 illustrates the effect of initial contention window size $\left(W_{0}\right)$ on ranging request collision probability when the number of CPEs are taken as $n=2,5,10$ and 20. At $W_{0}=2$, the ranging request collision probability, or simply collision probability, is too high. It is very hard for the CPEs to join the network in the first attempt. Ultimately, they will go in binary exponential random backoff (BERB) process. At $W_{0}=4$, the collision probability is 0.3672 for $n=$ 5. When number of CPEs approaches 10 , the collision probability becomes 0.7560 . This means when the number of CPEs is increased by $100 \%$, the collision probability is significantly increased by more than $100 \%$. Only a few CPEs can establish their association with the BS at this current initial contention window size.

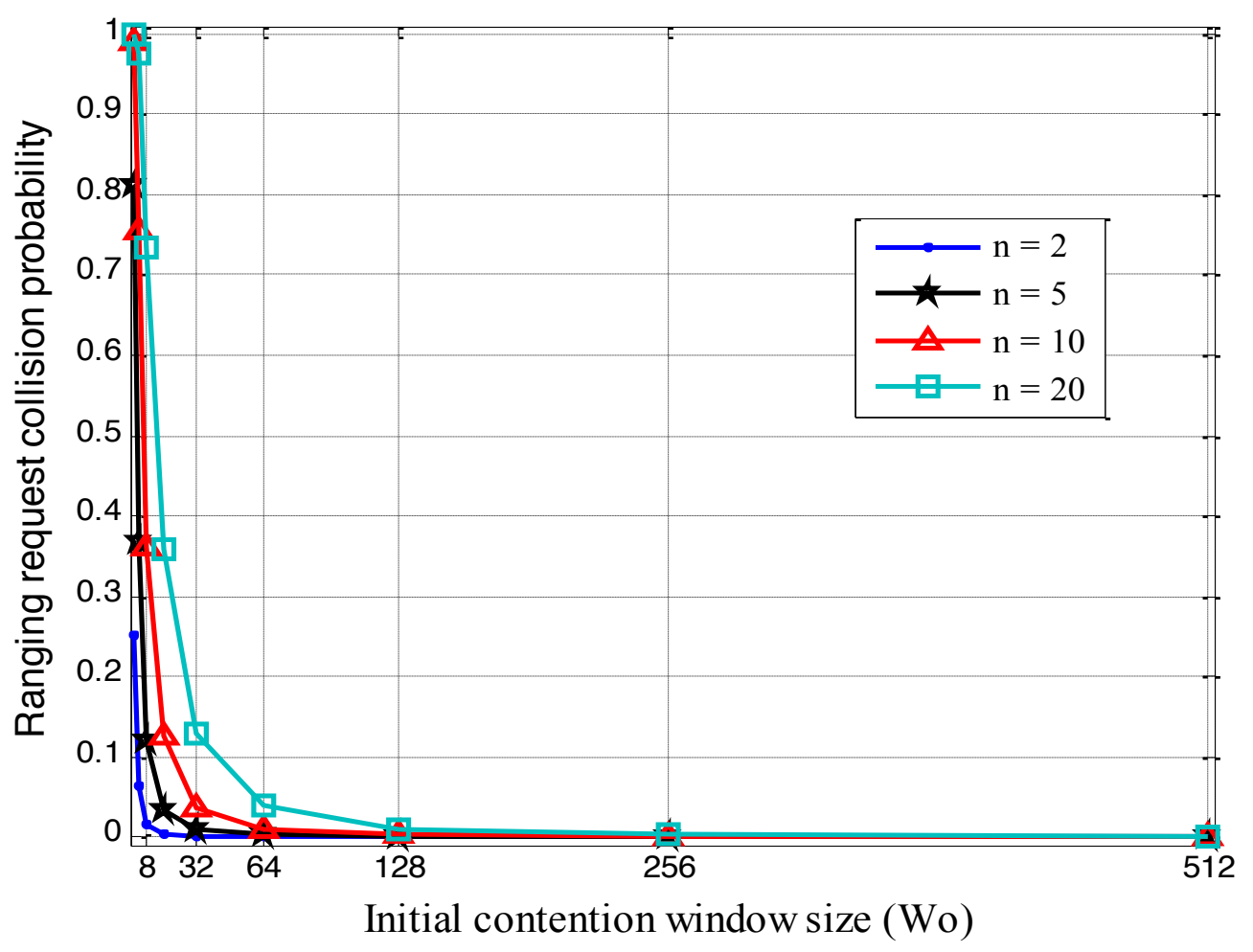

Figure 5: Ranging request collision probability versus initial contention window size.

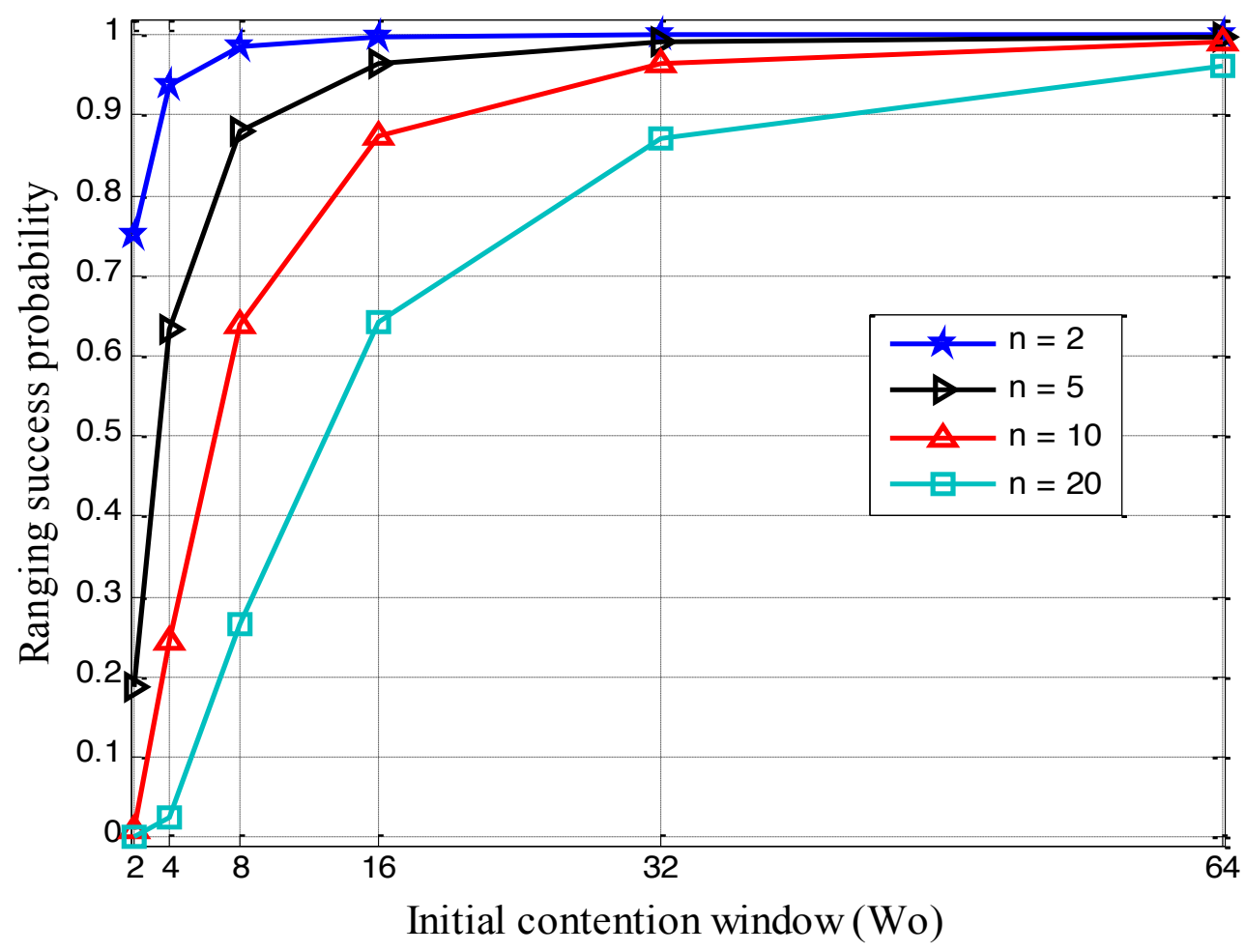

Figure 6: Ranging success probability versus initial contention window size. 
Similarly, when the number of CPEs is further increased by $100 \%$ from 10 to 20 , the respective collision probability turns into 0.9757 , which is a further $29 \%$ more from the previous one. In this case, the majority of the CPEs will be involved in the BERB procedure, which results in more delay. Now, at the other perspective, when $W_{0}$ is doubled from 4 to 8 , the collision probability is decreased by about $67 \%, 52 \%$ and $25 \%$ for $n=5, n=10$ and $n=20$ respectively. Obviously, the ranging success probability given in Figure 6 will be increasing. There are more chances for the CPEs to become part of the IEEE 802.22 network. Again, when $W_{0}$ is changed from 8 to 16 , the collision probability for $n=$ 5 rapidly approaches to zero. The collision probability for $n=10$ is further reduced by around $65 \%$. It is worth noting that in the case for $n=20$, the collision probability is drastically decreased by $51 \%$.

If we extend the window size upto 512 ( 9 bits) then we can see that from Figure 5, the collision probability exponentially decreases with increasing the window size. For example, at $W_{0}=32$, the collision probability for $n=20$ is 0.1281 and it is asymptotically converging to zero for higher values of $W_{0}$, which implies more CPEs can join the system. The same is true for other values of $n$.

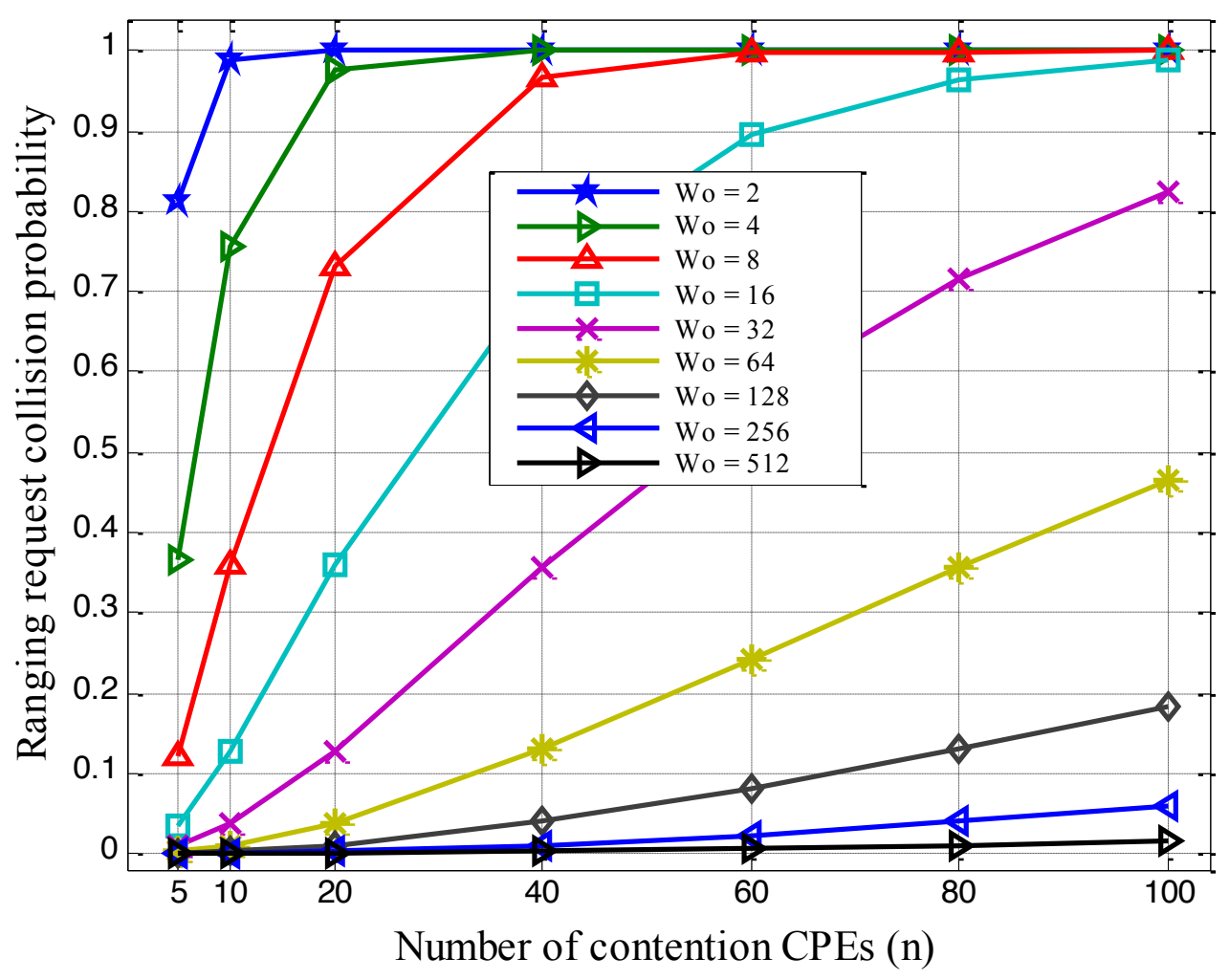

Figure 7: Ranging request collision probability versus number of contention CPEs over different initial contention window sizes.

To see the behavior of initial contention window size $W_{0}$ more precisely, we have plotted the ranging request collision probability and ranging success probability for $n=5,10,20,40,60,80,100$ in Figure 7 and Figure 8, respectively, over different values of $W_{0}$. In this experiment, it can be observed that at a particular value of $W_{0}$, as the number of contention CPEs increases, the collision probability goes on increasing. For the fixed value of $n$, while increasing the initial contention window size, the collision probability decreases.

Now we investigate the impact of initial contention window size on the performance in terms of average ranging success delay (Let us call it average delay, $D$ ). At $\mathrm{W}_{0}=4$, the average delay is almost less than 10 millisecond $(\mathrm{ms})$ for all contention CPEs, as shown in Figure 9 . When $\mathrm{W}_{0}$ is increased by $100 \%\left(\mathrm{~W}_{0}=8\right)$, the average delay for $n=5$ is increased by $51.40 \%$. At the same initial contention window size when $n$ is increased from 5 to $10, D$ is decreased by about $27 \%$. If $n$ is increased up to $20, D$ is further decreased by $58.21 \%$. The number of contention CPEs at this window size will experience less average delay to establish their associations with the BS. 


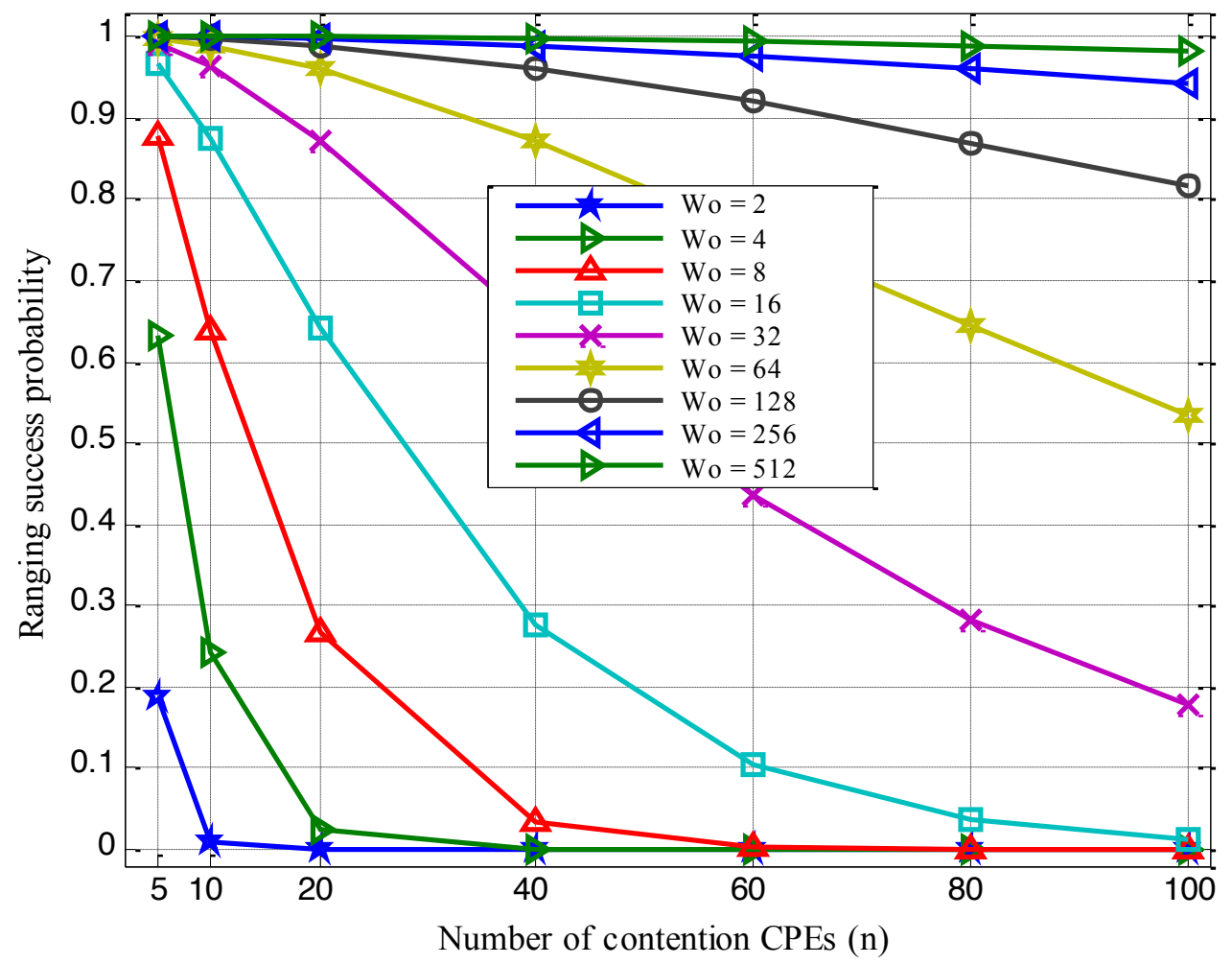

Figure 8: Ranging success probability versus number of contention CPEs over different initial contention window sizes.

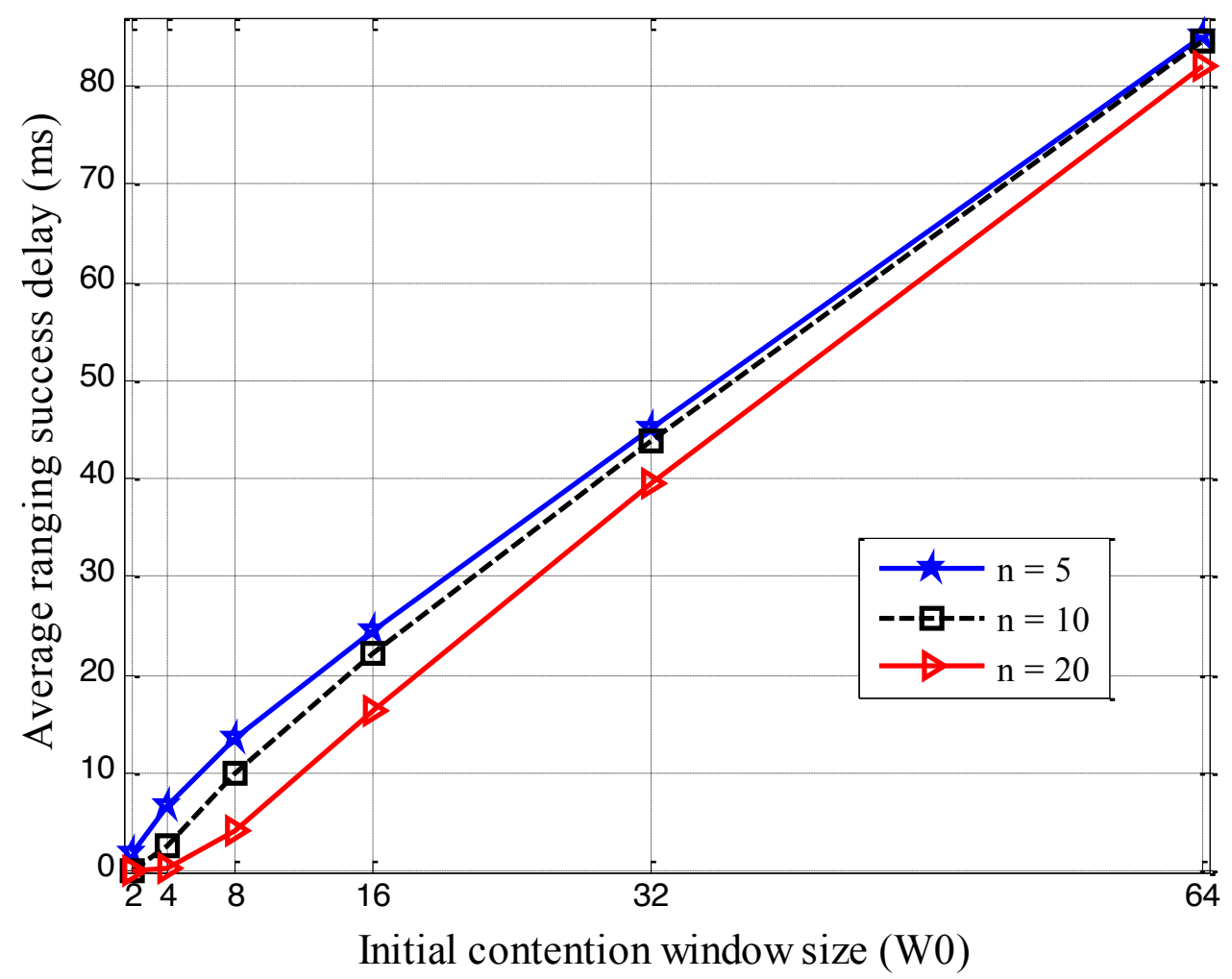

Figure 9: Average ranging success delay versus initial contention window size.

Again, when $\mathrm{W}_{0}$ is changed from 8 to 16 , the average delay is increased by $81.15 \%, 125.68 \%, 296.59 \%$ for $n=5, n=$ 10 and $n=20$, respectively. It is very clear from this figure, when window size is doubled, the average delay is significantly increased. In the case when initial contention window size is set to be 32 then average delay is more or less the same $(45 \mathrm{~ms})$, specifically when number of contention CPEs are up to 10. It means each CPE has to wait at least four frames duration on average before its association completion. Note that the frame duration in IEEE 802.22 network is $10 \mathrm{~ms}$. Now. similarlv if window size $\mathrm{W}_{\mathrm{n}}$ is moved from 32 to 64 . then the resulting average delav is 
increased by $89.40 \%, 93.40 \%$ and $107.46 \%$ for $n=5, n=10$ and $n=20$, respectively. The overall average delay is more than $80 \mathrm{~ms}$ for $\mathrm{n} \leq 20$. With this window size, each CPE needs to wait at least eight frames duration on average for its connection establishment with the BS, as it is evident from Figure 10. To reduce the average delay, the initial window size needs to be decreased. By doing this, the collision probability will be increased and ultimately, a fewer number of CPEs will establish their connection with the BS and many of them will be involved in the BERB process. Therefore, to keep the collision probability at a tolerable level to facilitate more CPEs establishing their connections with reasonable delay, it is necessary for the BS to schedule the optimized initial contention window size.

To test the validity of the expression obtained in Equ (18) and to find out the least upper bound on initial contention window size for the specific number of contention CPEs with $\mathrm{P}_{\mathrm{c}}(\mathrm{j})<\Omega$, we suppose $\Omega=0.1 \in[0,1], m=$ 0 and $n=2$, for $\mathrm{P}_{\mathrm{c}}(0)<0.1$, after using expression (16), we come up with $\mathrm{W}_{0}{ }^{2}>10 \Rightarrow \mathrm{W}_{0}>\sqrt{10}=3.16$, Therefore, $\mathrm{W}_{0} \geq 4$, which means, the minimum initial contention window size should be 4 ( 2 bits) to meet the requirements. Again, for $n=10$, and now the desired collision probability is $\mathrm{P}_{c}(0)<0.2$, using expression $(16)$, $\mathrm{W}_{0}{ }^{2}>155 \Rightarrow \mathrm{W}_{0}>\sqrt{155}=12.45$. This implies that $\mathrm{W}_{0} \geq 16$, the minimum initial contention window size in this case should be 16 (4 bits) to fulfill the desired specifications. These mathematical results can be verified in ranging request collision probability versus initial contention window size plot of Figure 4.

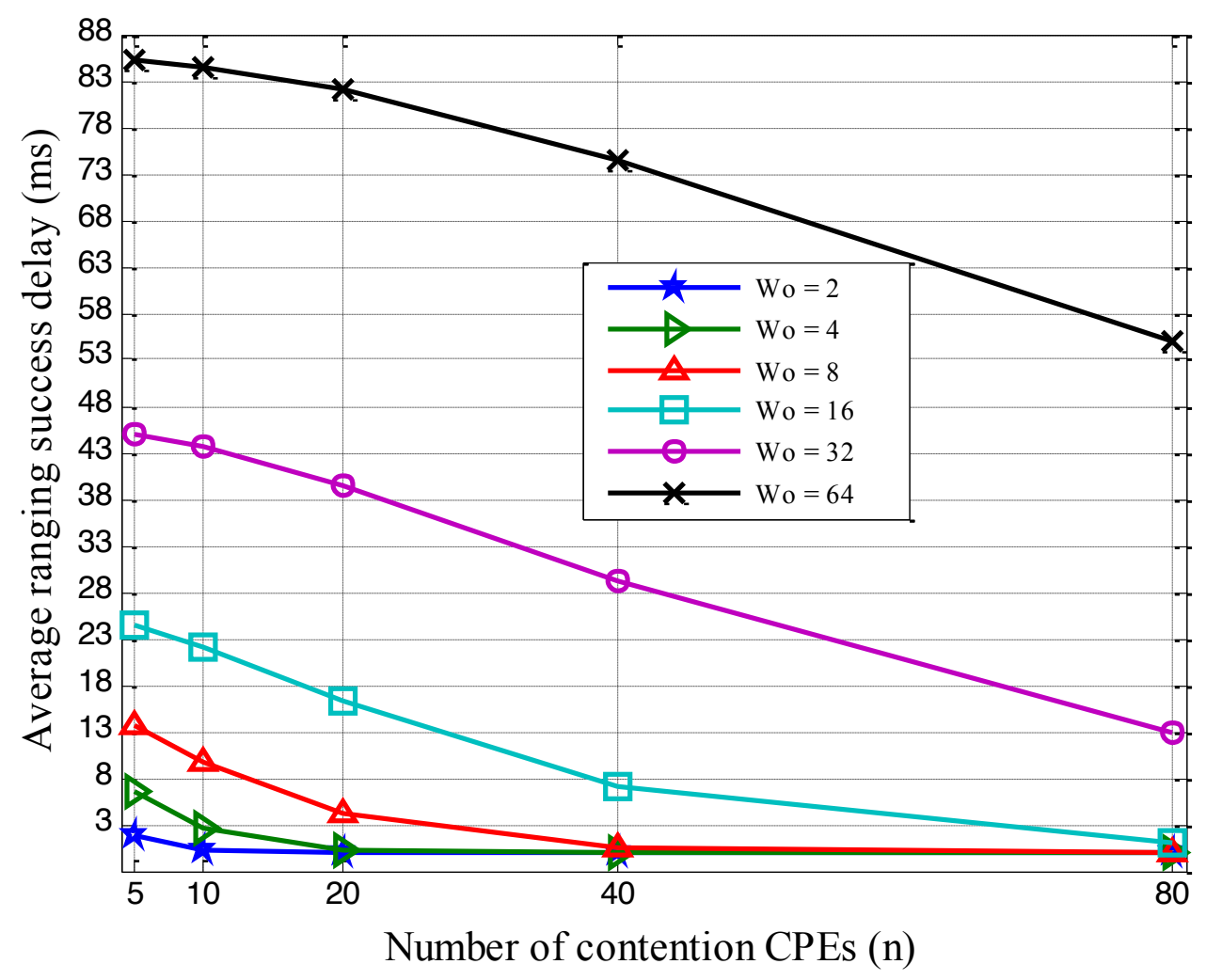

Figure 10: Average ranging success delay versus number of contention CPEs over different initial contention window sizes.

\section{Conclusion and future work}

In this paper, an analytical model has been derived to analyze the performance of initial ranging requests in a wireless network. The ranging request collision probability and ranging request success delay are investigated to evaluate the performance of the network. We have shown that the ranging success probability as well as average ranging delay, is strongly dependent upon the system parameters, mainly the initial contention window size and the number of contention CPEs in the network. To reduce the average delay, the initial window size needs to be decreased. By doing this, the ranging success probability will be decreased and, ultimately, fewer CPEs will establish their connection with the BS and many of them will be involved in binary exponential random backoff process. So to keep the collision probability at a tolerable value to facilitate more CPEs establishing their connections with less delay, it is necessary to optimize the initial contention window size, which the BS can schedule. This analysis can help the BS in scheduling the initial contention window size to facilitate the maximum number of contention CPEs to associate with reasonable delay leading to reach better system performance. 
Since continuous-time Markov chain model provides approximate solution, so in order to develop more realistic model for real type of networks, a discrete-time Markov chain model is essential. Our future work will focus on working this model for performance evaluation of CPEs attempting to associate with the BS in the IEEE 802.22 network.

\section{References}

[1] Federal Communications Commission, "Second Report and Order and Memorandum Opinion and Order", FCC 08-260, Nov. 2008.

[2] 802.22-2011 - IEEE Standard for Information Technology--Telecommunications and information exchange between systems Wireless Regional Area Networks (WRAN)--Specific requirements Part 22: Cognitive Wireless RAN Medium Access Control (MAC) and Physical Layer (PHY) Specifications: Policies and Procedures for Operation in the TV Bands, pp. 1-680, July 11, 2011.

[3] P802.22a/D1, IEEE Draft Standard for Information Technology--Telecommunications and information exchange between systems Wireless Regional Area Networks (WRAN)--Specific requirements Part 22: Cognitive Wireless RAN Medium Access Control (MAC) and Physical Layer (PHY) Specifications: Policies and Procedures for Operation in the TV Bands Amendment: Management and Control Plane Interfaces and Procedures and enhancement to the Management Information Base (MIB), pp. 1-551, September 10, 2013.

[4] Y. Chang-Jiang et al., "Study of RF Subsystem Used in Dynamic Spectrum Sharing System at TV Band", IEEE Transactions on Industrial Electronics, Vol. 60, No. 6, pp. 2346-2357, 2013.

[5] "Report of the spectrum efficiency working group", Federal Communications Commission, Washington, DC, USA, Tech. Rep. 02-155, Nov. 2002.

[6] M. Nekovee, "A Survey of Cognitive Radio Access to TV White Spaces", International Journal of Digital Multimedia Broadcasting, Vol. 2010, pp: 1-12, 2010.

[7] J. V. Beek, J. Riihijarvi, A. Achtzehn, P. Mahonen. "TV white space in Europe", IEEE Transactions on Mobile Computing, Vol.11, No. 2, pp. 178-188, 2012.

[8] V. Mishra, C. T. Lau, S. Chan, "Reconfigurable Channel Selection Technique for Cognitive Radio Network with Heterogeneous Primary Bands", IEEE Transactions on Vehicular Technology, Vol. 62, No. 8, pp. 3942-3952, 2013.

[9] H. Elshafie, , N. Fisal, M. Abbas, W. A. Hassan, H. Mohamad, N. Ramli, S. Jayavalan, and S. Zubair "A survey of cognitive radio and TV white spaces in Malaysia", Transactions on Emerging Telecommunications Technologies, pp. 1-17, 2014.

[10] Y.-C. Liang, A. T. Hoang, H.-H. Chen, "Cognitive radio on TV bands: A new approach to provide wireless connectivity for rural areas”, IEEE Wireless Communication, vol. 15, no. 3, pp. 16-22, Jun. 2008.

[11] F. Li, P. Shi, L. Wu, M.V Basin., C.C. Lim "Quantized control design for cognitive radio networks modeled as nonlinear semi-Markovian jump systems”, IEEE Transactions on Industrial Electronics, accepted, 26 July 2014.

[12] C. Cordeiro, K. Challapali, D. Birru, "IEEE 802.22: An Introduction to the First Wireless Standard based on Cognitive Radios", Journal of Communications, Vol. 1, No. 1, April 2006.

[13] M. T. Masonta, M. Mzyece, N. Ntlatlapa, "Spectrum Decision in Cognitive Radio Networks: A Survey", IEEE Communications Surveys \& Tutorials, Vol. 15, No. 3, pp. 1088-1107, 2013.

[14] Y. S. Hsieh, K. C. Wang, Chun-Ting Chou, T. Y. Hsu, Tu-I Tsai, Y. S. Chen, "Quiet Period (QP) Scheduling Across Heterogeneous Dynamic Spectrum Access (DSA)-Based Systems”, IEEE Transactions on Wireless Communications, Vol. 11, No. 8, pp. 2796-2805, 2012.

[15] M. Youssef, M. Ibrahim, M. Abdelatif, L. C., Athanasios V. Vasilakos, "Routing Metrics of Cognitive Radio Networks: A Survey”, IEEE Communications Surveys \& Tutorials, Vol. 16, No. 1, pp. 92-109, 2014.

[16] L.-C. Wang, C-W Wang, C.-J. Chang," Modeling and Analysis for Spectrum Handoffs in Cognitive Radio Networks", IEEE Transactions on Mobile Computing, Vol. 11, No. 9, pp. 1499-1513, September 2012.

[17] H. Kim, K. G. Shin, "In-Band Spectrum Sensing in IEEE 802.22 WRANs for Incumbent Protection", IEEE Transactions on Mobile Computing, Vol. 9, No. 12, pp. 1766-1779, December 2010.

[18] A. W. Min, K. G. Shin, X. Hu, "Secure Cooperative Sensing in IEEE 802.22 WRANs Using Shadow Fading Correlation", IEEE Transactions on Mobile Computing, Vol. 10, No. 10, pp. 1434-1447, October 2011.

[19] M. Morelli, L. Sanguinetti, H. V. Poor, “A Robust Ranging Scheme for OFDMA-Based Networks”, IEEE Transactions on Communications, Vol. 57, No. 8, pp. 2441-2452, 2009.

[20] R. Miao, L. Gui, J. Sun, J. Xiong, “A Ranging Method for OFDMA Uplink System”, IEEE Transactions on Consumer Electronics, Vol. 56, No. 3, pp. 1223-1228, 2010.

[21] Y.-H. Liang, B.-J. Chang, S.-J. Hsieh, D.-Y. Wang, “Analytical Model of QoS-Based Fast Seamless Handoff in IEEE 802.16j WiMAX Networks", IEEE Transactions on Vehicular Technology, Vol. 59, No. 7, pp. 3549-3561, 2010.

[22] L. Sanguinetti, M. Morelli, “An Initial Ranging Scheme for the IEEE 802.16 OFDMA Uplink”, IEEE Transactions on Wireless Communications, Vol. 11, No. 9, pp. 3204-3215, 2012. 
[23] H. Afzal, I. Awan, M. R. Mufti, R. E. Sheriff, "Modeling and Analysis of Customer Premise Equipments Registration Process in IEEE 802.22 WRAN Cell”, Journal of Systems and Software (Elsevier Journal), Vol. 98, pp. 107-116, August 2014.

[24] H. Afzal, I. Awan, M. R. Mufti, R. E. Sheriff, "Modeling of initial contention window size for successful initial ranging process in IEEE 802.22 WRAN cell”, Simulation Modelling Practice and Theory (Elsevier Journal), Vol. 51, pp. 135-148, 2015.

[25] H. Afzal, I. Awan, M. R. Mufti, "Mathematical Modeling of Association Attempt with the Base Station for Maximum Number of Customer Premise Equipments in the IEEE 802.22 Network", Proc. in IEEE, $3^{\text {rd }}$ International Conference on Future Internet of Things and Cloud (FiCloud), Italy, Rome, pp. 289-292, 2015. 